\title{
Efecto del esfuerzo de muestreo en la riqueza de táxones de macroinvertebrados acuáticos y el índice BMWP/Atitlán
}

\author{
Fátima Reyes-Morales ${ }^{1} \&$ Monika Springer ${ }^{1,2}$ \\ 1. Escuela de Biología, Universidad de Costa Rica, 2060 San Pedro de Montes de Oca, San José, Costa Rica; \\ fatimarys3@gmail.com \\ 2. Centro de Investigación en Ciencias del Mar y Limnología (CIMAR), Universidad de Costa Rica, 2060 San Pedro de \\ Montes de Oca, San José, Costa Rica; monika.springer@ucr.ac.cr
}

Recibido 12-XII-2013. C Corregido 20-I-2014. Aceptado 13-II-2014.

\begin{abstract}
Effect of sampling effort on taxa richness of aquatic macroinvertebrates and the BMWP/Atitlán index. Aquatic macroinvertebrates are the group of organisms most commonly used to determine ecosystem health in water quality studies and freshwater biomonitoring. Nevertheless, the methods and collecting time of biomonitoring have not yet been sufficiently adapted and tested in tropical aquatic environments. Twelve rivers in the Lago de Atitlán watershed in Guatemala were assessed with different collecting times, during the dry season. The method involved the collection of organic and inorganic material including benthic organisms, from different microhabitats, for a pre-established time period $(5,10,15 \mathrm{~min})$ with a $\mathrm{D}$-frame net. Samples were preserved with $95 \%$ ethanol in the field, and sorted in the laboratory. As expected, the analysis showed that the abundance and taxonomic richness was higher with increasing sampling effort. The water quality categories obtained from the newly proposed BMWP/Atitlán index varied among sampling times. However, the KruskalWallis test showed no significant differences between the categories obtained with the index and the number of taxa collected at 10 and $15 \mathrm{~min}$. Therefore, we recommend a reduction of sample time, but maintaining the tree subsamples in order to include most variety of microhabitats and assure a representative sample of the aquatic macroinvertebrates. Rev. Biol. Trop. 62 (Suppl. 2): 291-301. Epub 2014 April 01.
\end{abstract}

Key words: freshwater biomonitoring, aquatic macroinvertebrates, water quality, Lago de Atitlán, Guatemala.

En los últimos años diversos cuerpos de agua de Guatemala, incluyendo los de la cuenca del lago de Atitlán, se han visto influenciados y hasta amenazados por diversas actividades humanas, incrementado de manera considerable el deterioro del ecosistema y la calidad del agua (Castellanos, Girón, Álvarez, López \& España, 2002; CONAP, 2007). Tales impactos demandan la implementación de metodologías innovadoras para su preservación y conservación. Los análisis fisicoquímicos han sido el método más utilizado durante décadas para evaluar la calidad de las aguas. No obstante, estos parámetros son ineficaces para detectar cambios o alteraciones en el ambiente en períodos más prolongados, por lo que es necesario integrarlos con métodos biológicos, para que en conjunto reflejen la salud integral del ecosistema acuático y poder plantear planes de manejo adecuados. El uso de métodos biológicos en el monitoreo de cuerpos de agua se ha convertido en una herramienta valiosa y complementaria de los métodos físicoquímicos y bacteriológicos (Johnson, Wiederholm \& Rosenberg, 1993; Barbour, Gerritsen, Snyder \& Stribling, 1999; Sermeño-Chicas et al., 2010a; Springer, 2010).

Los macroinvertebrados acuáticos se han utilizado en muchos países como bioindicadores para evaluar el estado de salud de los ríos; no obstante estos métodos no reemplazan el tradicional método de los análisis fisicoquímicos. Un bioindicador es un organismo que ha sido seleccionado debido a que muestra sensibilidad o tolerancia a diversos 
tipos de contaminación o sus efectos (Resh \& Jackson, 1993; Rosenberg \& Resh, 1993). Existen numerosos protocolos de muestreo de macroinvertebrados acuáticos e índice bióticos utilizados en diversos países para evaluar y determinar el estado de salud de los ríos (Resh, Norris \& Barbour, 1995; Barbour et al., 1999; Bonada et al., 2002; Bonada, Dallas, Rieradevall, Prat \& Day, 2006). Estos protocolos han sido diseñados para que su implementación sea fácil, de bajo costo y sobre todo poder ser aplicados en diversas regiones y estudios.

En Centroamérica se realizan cada vez más estudios utilizando macroinvertebrados acuáticos como indicadores biológicos (Sermeño et al., 2010a; Springer, 2010; entre otros). Sin embargo, hay pocos antecedentes sobre la aplicación de índices bióticos en Guatemala, aun cuando se ha demostrado total eficacia en la detección de alteración en la calidad del agua en otras regiones. Reyes-Morales (2012) recientemente adaptó y validó el índice biótico BMWP (Biological Monitoring Working Party) para evaluar la calidad de los cuerpos de agua de la cuenca de Atitlán, Guatemala.

Para una correcta aplicación de estos índices es importante contar con métodos de muestreos estandarizados. Existen pocos estudios sobre el efecto del esfuerzo de muestreo y método de recolecta en países de Centroamérica (Paaby, Ramírez \& Pringle, 1998; Maue \& Springer, 2008; Stein, Springer \& Kohlmann, 2008). Algunos de estos estudios han demostrado que los resultados en cuanto a estructura y composición taxonómica de la comunidad pueden variar de acuerdo al método empleado. También se ha determinado que las categorías de calidad del agua varían según el método y el esfuerzo de recolecta. Esto hace evidente la necesidad de calibrar los métodos de muestreo a utilizar en estudios de biomonitoreo acuático y la aplicación de índices bióticos. El objetivo principal del presente estudio fue evaluar el efecto del esfuerzo de muestreo sobre la riqueza de táxones, abundancia de macroinvertebrados acuáticos y especialmente el índice BMWP, adaptado para la cuenca del Lago Atitlán (Reyes-Morales, 2012) y así establecer el método y el esfuerzo de recolecta más apropiado para el índice.

\section{MATERIALES Y MÉTODOS}

Área de estudio: La Cuenca del Lago de Atitlán es un sistema endorreico que se encuentra ubicado en el altiplano de Guatemala $\left(14^{\circ} 36^{\prime}-14^{\circ} 44^{\prime} \mathrm{N}, 91^{\circ} 07^{\prime}-91^{\circ} 17^{\prime} \mathrm{W}\right)$. La cuenca tiene un área de $541 \mathrm{~km}^{2}$, la elevación oscila entre los 1562 y $3587 \mathrm{~m}$ snm. Los dos principales ríos permanentes son el río Quiscab y el río Panajachel. La cuenca del Lago de Atitlán pertenece a la unidad biogeográfica de bosque de montaña y a la ecorregión de bosque de pinoencino de Centroamérica (CONAP, 2008). Las principales actividades que se desarrollan en la cuenca son la agricultura, ecoturismo, agroforestales, pesquera y urbano-industrial (Castellanos et al., 2002). El estudio se realizó en doce sitios de muestreo (Fig. 1), ubicados en tres diferentes usos del suelo (cultivos, bosque y poblados) y a diferentes altitudes para incluir la mayor variabilidad altitudinal dentro de la cuenca (Cuadro 1) (Reyes-Morales, 2012).

Macroinvertebrados acuáticos: Las muestras se recolectaron en los mismos sitios que se describen en el Cuadro 1 en dos fechas (Febrero y Abril, 2011) y durante la época seca para minimizar la variación temporal de la distribución de los macroinvertebrados acuáticos. La recolecta se realizó mediante la metodología de muestreo multi-hábitats (e.g., piedra, hojarasca, troncos, arenas), con una red tipo "D" con una abertura de malla de $500 \mu \mathrm{m}$. Se recolectaron tres muestras de $5 \mathrm{~min}$ cada una por sitio (MINAE-S, 2007; Sermeño et al., 2010b). El material recolectado se preservó en etanol al 95\%, para su posterior separación y clasificación en el laboratorio. Los organismos recolectados fueron identificados en su mayoría a género, con claves taxonómicas apropiadas para la región (Merritt, Cummins \& Berg, 2008; Springer, Ramírez \& Hanson, 2010). El material identificado se encuentra depositado en la colección de insectos acuáticos del Museo de Zoología de la Universidad de Costa Rica 


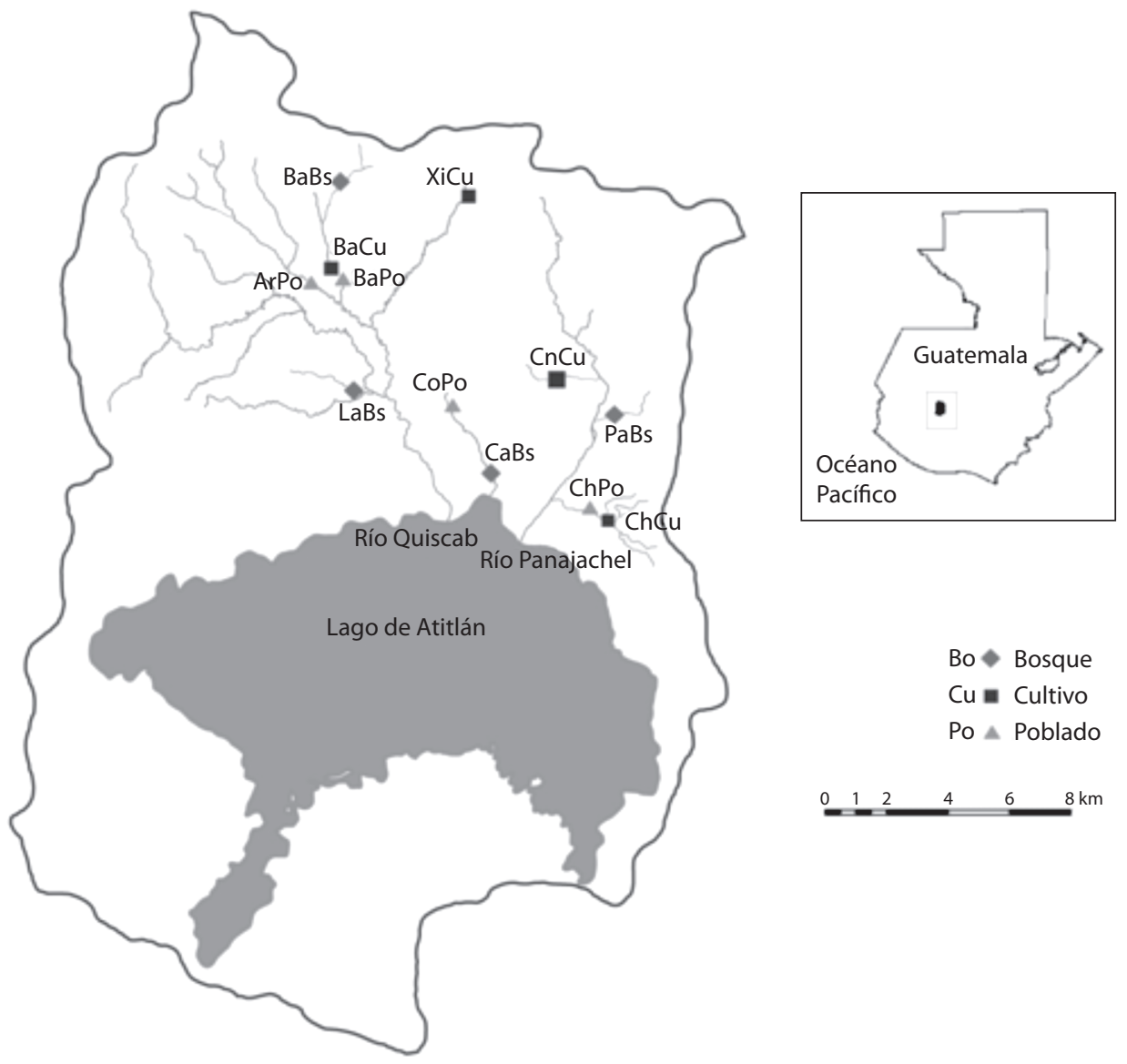

Fig. 1. Ubicación de la cuenca dentro del territorio de Guatemala y de los sitios de muestreo en la Cuenca del Lago de Atitlán. (Ar) Argueta, (Ba) Barreneché, (Ca) Catarata, (Ch) Chicansanres, (Co) Cojolyá, (Cn) Concepción, (La) Labor, (Pa) Panasacar y (Xi) Xibalbay.

Fig. 1. Location of the watershed within the territory of Guatemala and sampling sites in the Lago Atitlan watershed. (Ar) Argueta, (Ba) Barreneché, (Ca) Catarata, (Ch) Chicansanres, (Co) Cojolyá, (Cn) Concepción, (La) Labor, (Pa) Panasacar y (Xi) Xibalbay.

(MZUCR) y una colección de referencia en la Colección de Artrópodos de la Universidad del Valle de Guatemala (UVG).

Análisis de datos: Se calcularon los valores de calidad del agua con el índice BMWP modificado y adaptado para la cuenca del Lago de Atitlán (BMWP/Atitlán) (Reyes-Morales, 2012), basado en el índice biótico BMWPCR (MINAE-S, 2007). Este se calcula con la suma de los valores de tolerancia asignadas a las familias de macroinvertebrados presentes en la muestra. El índice se calculó tres veces para determinar la variación de las categorías según el esfuerzo de muestreo (5, 10 y $15 \mathrm{~min})$. Para generar muestras de 5,10 y $15 \mathrm{~min}$ se combinaron muestras individuales de $5 \mathrm{~min}$ en secuencia. Se realizó un análisis de varianza no paramétrico (Kruskal-Wallis) (Quinn \& Keough, 2003), para evaluar si el esfuerzo de muestreo tuvo un efecto significativo en el número de táxones registrados y en las categorías de los índices bióticos calculados. Los datos fueron analizados con el programa Statistix 8.0. 
CUADRO 1

Características de los sitios de muestreo en la cuenca del Lago de Atitlán, Guatemala, 2011.

(Ver Fig. 1 para acrónimos de los sitios).

TABLE 1

Characteristics of the sampling sites in the Lago Atitlan watershed, Guatemala, 2011. (See Fig. 1 for acronyms of the sites).

\begin{tabular}{cccccc}
\multirow{3}{*}{ Uso del suelo } & Localidad & Altitud & Ancho & Profundidad & Velocidad del agua \\
Bosque & $\mathrm{msnm}$ & $(\mathrm{m})$ & Máxima $(\mathrm{m})$ & $(\mathrm{m} / \mathrm{s})$ \\
& $\mathrm{BaBs}$ & 2548 & $0.7-1.2$ & 0.16 & $0.19 \pm 0.15$ \\
& $\mathrm{CaBs}$ & 1689 & $1.7-2.6$ & 0.26 & $0.22 \pm 0.09$ \\
\multirow{5}{*}{ Cultivo } & $\mathrm{LaBs}$ & 2200 & $1.0-1.8$ & 0.16 & $0.2 \pm 0.05$ \\
& $\mathrm{PaBs}$ & 1779 & $0.9-1.3$ & 0.23 & $0.55 \pm 0.27$ \\
& $\mathrm{BaCu}$ & 2305 & $0.5-2.6$ & 0.19 & $0.43 \pm 0.45$ \\
\multirow{5}{*}{ Poblado } & $\mathrm{ChCu}$ & 1980 & $1.7-1.9$ & 0.24 & $0.71 \pm 0.06$ \\
& $\mathrm{CnCu}$ & 2047 & $0.2-0.7$ & 0.15 & $0.26 \pm 0.05$ \\
& $\mathrm{XiCu}$ & 2364 & $3.0-3.5$ & 0.19 & $0.4 \pm 0.02$ \\
& $\mathrm{ArPo}$ & 2301 & $4.0-4.6$ & 0.30 & $0.61 \pm 0.03$ \\
& $\mathrm{BaPo}$ & 2293 & $1.9-3.8$ & 0.30 & $0.54 \pm 0.12$ \\
& $\mathrm{ChPo}$ & 1946 & $2.2-2.8$ & 0.46 & $0.27 \pm 0.07$ \\
\hline
\end{tabular}

\section{RESULTADOS}

Abundancia y riqueza de táxones: $\mathrm{Se}$ recolectaron un total de 47482 individuos de macroinvertebrados acuáticos, pertenecientes 54 familias y 16 órdenes (Cuadro 2). En la primera fecha se recolectaron 23241 individuos y 24241 en la segunda fecha. Los grupos más diversos y abundantes fueron Diptera, Trichoptera, Ephemeroptera y Coleoptera. Las familias con mayor abundancia y frecuencia fueron Chironomidae, Simuliidae (Diptera) y Baetidae (Ephemeroptera). La cantidad de individuos recolectados aumentó al incrementar el esfuerzo de muestreo (Fig. 2). Los sitios con las mayores abundancias de individuos durante ambos muestreos fueron los localizados en bosque, seguido de poblado.

En el primer muestreo se registraron 68 táxones (48 familias, 14 órdenes/grupos), mientras que en el segundo muestreo 71 táxones de los cuales 16 táxones (9 familias, 2 órdenes/ grupos) no fueron recolectados en el primer periodo de muestreo. En general, la riqueza de táxones incrementó al aumentar el esfuerzo de recolecta, en cada tipo de río y fecha de muestreo (Fig. 3). Sin embargo, algunos sitios mantuvieron una riqueza de táxones similar entre un tiempo de esfuerzo y el siguiente, este patrón fue observado en todas las categorías de uso de terreno.

El análisis de Kruskal-Wallis reveló que no existe una diferencia significativa entre el número de individuos de macroinvertebrados recolectados y el número de táxones registrado con un esfuerzo de 10 y $15 \mathrm{~min}$, en ambas fechas $(p>0.05)$. Solo se encontraron diferencias significativas entre $5-10 \mathrm{~min}$ y $5-15 \mathrm{~min}$ de esfuerzo (Kruskal-Wallis: $\mathrm{p}<0.05$ ).

Valor del índice BMWP: Las categorías obtenidas del cálculo del índice BMWP/Atitlán por esfuerzo de muestreo variaron entre los diferentes tiempos de muestreo y condiciones del uso del suelo (Fig. 4). Durante el primer muestreo el río Chicansanres (cultivo) y el río Argueta (poblado) fueron los sitios que mostraron la mayor variación (tres niveles) en cuanto a las categorías obtenidas. En el mes de abril solo el sitio del río Concepción (cultivo) fue el que mostró una variación de tres categorías. El resto de los sitios en ambas fechas se 
CUADRO 2

Listado taxonómico y abundancias de macroinvertebrados acuáticos recolectados en ambas fechas en los ríos de la cuenca del Lago de Atitlán, Guatemala. (Ver Fig.1 para siglas de los sitios).

TABLE 2

Taxonomic list and abundances of aquatic macroinvertebrates collected on both dates in the streams of the Lago Atitlan watershed, Guatemala. (See Fig.1 for acronyms of the sites).

\begin{tabular}{|c|c|c|c|c|c|c|c|c|c|c|c|c|}
\hline \multirow{2}{*}{ Orden/Familias } & \multicolumn{4}{|c|}{ Bosque } & \multicolumn{4}{|c|}{ Cultivo } & \multicolumn{4}{|c|}{ Poblado } \\
\hline & $\mathrm{Ba}$ & $\mathrm{Ca}$ & $\mathrm{La}$ & $\mathrm{Pa}$ & $\mathrm{Ba}$ & $\mathrm{Ch}$ & $\mathrm{Co}$ & $\mathrm{Xi}$ & $\mathrm{Ar}$ & $\mathrm{Ba}$ & $\mathrm{Ch}$ & Co \\
\hline \multicolumn{13}{|l|}{ Diptera } \\
\hline Ceratopogonidae & 13 & 2 & 7 & 8 & & & 1 & & & & & \\
\hline Chironomidae & 4349 & 708 & 1423 & 291 & 939 & 11 & 367 & 362 & 1130 & 3844 & 842 & 2883 \\
\hline Psychodidae & 1 & 218 & 2 & 8 & & & 11 & & 1 & 265 & 30 & 133 \\
\hline Tipulidae & 81 & 14 & 47 & 32 & 9 & 2 & 15 & 6 & 8 & 9 & & 14 \\
\hline Simuliidae & 142 & 3355 & 669 & 2992 & 682 & 463 & 316 & 8 & 1708 & 908 & 21 & 38 \\
\hline Dixidae & 11 & & 3 & 1 & & & & & & & & \\
\hline Culicidae & & & & & & & & & 54 & & & \\
\hline Dolichopodidae & & & & 3 & & & & & 1 & 2 & & \\
\hline Empididae & 140 & 17 & 37 & 22 & 4 & & & 1 & 9 & 14 & & 3 \\
\hline Ephydridae & 1 & & 1 & 1 & & & & & 6 & 4 & & \\
\hline Muscidae & 1 & 2 & 3 & 4 & 4 & 1 & 9 & 7 & 66 & 27 & & 4 \\
\hline Ptychopteridae & & & 1 & & & & & & & & & \\
\hline Stratiomyidae & & 5 & & 38 & & 1 & & & & & & \\
\hline Syrphidae & & 1 & & & & & & & & & 1 & \\
\hline Tabanidae & & & & & & & 1 & & & & & 1 \\
\hline \multicolumn{13}{|l|}{ Trichoptera } \\
\hline Hydroptilidae & 2 & 2 & & 35 & & & 4 & & 4 & 4 & & \\
\hline Glossosomatidae & 1 & 11 & 1 & 16 & 2 & & & & 3 & & & \\
\hline Hydropsychidae & 222 & 213 & 356 & 42 & 359 & 7 & & & 6 & 63 & & \\
\hline Helicopsychidae & & 1 & & 1 & & & & & & & & \\
\hline Hydrobiosidae & 25 & 10 & 139 & 51 & 3 & 3 & 1 & & & & & \\
\hline Lepidostomatidae & 148 & 13 & 256 & 45 & 15 & & & & 1 & 30 & & \\
\hline Limnephilidae & & & & & 5 & & & 1 & 1 & 6 & & \\
\hline Philopotamidae & & & 61 & 1 & & & & & & & & \\
\hline Polycentropodidae & & & 2 & & & & & & & & & \\
\hline \multicolumn{13}{|l|}{ Coleoptera } \\
\hline Dytiscidae & 3 & 30 & 1 & 7 & 1 & 1 & & 1 & 13 & 7 & 1 & 7 \\
\hline Elmidae & & 1 & 1 & 1 & & & & & 1 & & & \\
\hline Hydrophilidae & 2 & 1 & 4 & & & & & & & & & \\
\hline Georissidae & & & & & & 1 & & & & & & \\
\hline Hydraenidae & & 1 & & & & & & & & & & \\
\hline Ptilodactylidae & & & & 1 & & 1 & & & & & & \\
\hline Scirtidae & & & 34 & & & 1 & & & & & & \\
\hline Curculionidae & 1 & & 1 & & & & & & & & & \\
\hline Staphylinidae & 48 & & 7 & 6 & 2 & & 1 & & & 1 & & 1 \\
\hline \multicolumn{13}{|l|}{ Ephemeroptera } \\
\hline Baetidae & 601 & 748 & 1634 & 2361 & 523 & 32 & 18 & 415 & 629 & 415 & 6 & 1492 \\
\hline Leptohyphidae & 64 & & & & & & & & & & & \\
\hline Leptophlebiidae & 18 & & & & & & & & & & & \\
\hline
\end{tabular}


CUADRO 2 (Continuación) / TABLE 2 (Continued)

\begin{tabular}{|c|c|c|c|c|c|c|c|c|c|c|c|c|}
\hline \multirow{2}{*}{ Orden/Familias } & \multicolumn{4}{|c|}{ Bosque } & \multicolumn{4}{|c|}{ Cultivo } & \multicolumn{4}{|c|}{ Poblado } \\
\hline & $\mathrm{Ba}$ & $\mathrm{Ca}$ & $\mathrm{La}$ & $\mathrm{Pa}$ & $\mathrm{Ba}$ & $\mathrm{Ch}$ & Co & $\mathrm{Xi}$ & $\mathrm{Ar}$ & $\mathrm{Ba}$ & $\mathrm{Ch}$ & Co \\
\hline \multicolumn{13}{|l|}{ Hemiptera } \\
\hline Gerridae & 3 & & & & & & & & & & & \\
\hline Mesoveliidae & & & & & & & & 1 & & 2 & & \\
\hline Veliidae & 5 & & 20 & 9 & & 87 & 15 & & & & 31 & \\
\hline \multicolumn{13}{|l|}{ Odonata } \\
\hline Aeshnidae & 7 & & 2 & & 1 & & & & & & & \\
\hline Calopterygidae & 3 & & & & 26 & & & & & 1 & & \\
\hline Coenagrionidae & 3 & & & & 5 & & & & & 1 & & \\
\hline Cordulegastridae & 1 & & & & & & & & & & & \\
\hline \multicolumn{13}{|l|}{ Lepidoptera } \\
\hline Crambidae & & 2 & 9 & 3 & & & 2 & & & & & \\
\hline \multicolumn{13}{|l|}{ Haplotaxida } \\
\hline Haplotaxidae & & 4 & & & & & & & & 11 & 408 & 124 \\
\hline Naididae & 2 & 5 & 17 & & 6 & 1 & & 575 & 588 & 36 & & 558 \\
\hline Tubificidae & 2 & & & & 3 & & 9 & 1 & 6 & & 12 & 65 \\
\hline \multicolumn{13}{|l|}{ Basommatophora } \\
\hline Ancyliidae & & & & & & & 3 & & & & & \\
\hline Physidae & & 1 & & & 1 & & 2631 & 3 & 1 & & & \\
\hline Planorbidae & & & & 1 & & & & & & & & \\
\hline \multicolumn{13}{|l|}{ Trombidiformes } \\
\hline Hydrachnidae & 56 & 32 & 22 & & & 1 & & & & & & 1 \\
\hline \multicolumn{13}{|l|}{ Decapoda } \\
\hline Pseudothelphusidae & & & & & & & 1 & & & & & \\
\hline \multicolumn{13}{|l|}{ Rhynchobdellida } \\
\hline Glossiphoniidae & & 1 & & & & & & & & & & \\
\hline \multicolumn{13}{|l|}{ Veneroida } \\
\hline Pisidiidae & 1 & & & & 1 & & 6 & & & & & \\
\hline Collembola & 13 & & 4 & & 3 & & 3 & & 2 & 1 & & \\
\hline Nemata & & & & & 1 & & & & & & & \\
\hline Turbellaria & 10 & 28 & 208 & & 549 & & & 1 & 2 & 4 & & \\
\hline
\end{tabular}

mantuvieron en la mayoría de los casos dentro de la misma categoría, a pesar de pequeñas variaciones del valor del índice BMWP/Atitlán. El análisis de Kruskal-Wallis realizado reveló que no existe una diferencia significativa en el puntaje obtenido en el índice BMWP entre los 5 y $10 \mathrm{~min}$ y entre los 10 y $15 \mathrm{~min}$ de esfuerzo de muestreo ( $p>0.05$ ). Sin embargo, si se observaron diferencias en el puntaje entre el esfuerzo de muestreo de los $5 \mathrm{~min}$ y los $15 \mathrm{~min}$ $(\mathrm{p}<0.05)$.

\section{DISCUSIÓN}

La riqueza de táxones es una importante expresión de la complejidad de la comunidad de macroinvertebrados acuáticos. En estudios que evalúan la calidad del agua se utiliza 


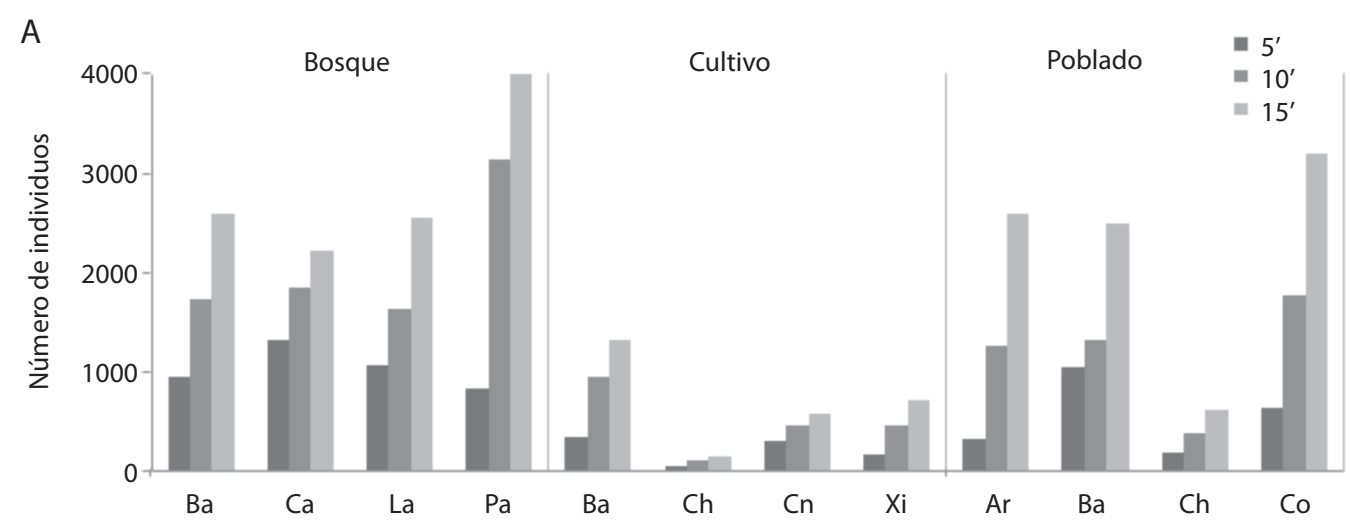

B

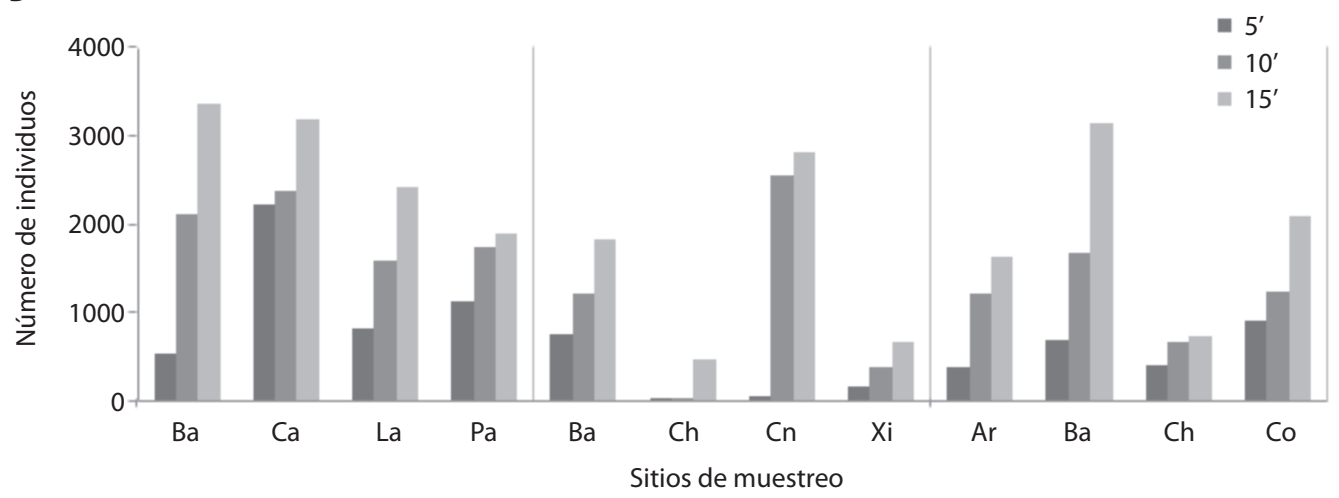

Fig. 2. Número total de individuos recolectados según el esfuerzo de muestreo en la cuenca del Lago de Atitlán, Guatemala. (A) Febrero y (B) Abril. (Ver Fig.1 para acrónimos de los sitios).

Fig. 2. Total number of individuals collected by the sampling effort in the Lago Atitlán watershed, Guatemala. (A) February and (B) April. (See Fig.1 for acronyms of the sites).

esta medida para hacer comparaciones entre sitios, con el fin de contestar a la pregunta si las comunidades difieren entre sí (e.g., sitios a evaluar vs sitios de referencia) (Courtemanch, 1996; Vinson \& Hawkins, 1996; Barbour et al., 1999; Bonada et al., 2002). En este estudio el que exista una diferencia entre la riqueza de táxones y la abundancia de macroinvertebrados acuáticos según el esfuerzo de muestreo, puede ser explicado por dos razones principales.

Primero, a mayor tiempo de recolecta mayor es el área muestreada, y por consiguiente se incluyen una mayor diversidad de sustratos o microhabitats. Los táxones en una comunidad se distribuyen en proporciones desiguales (Williams \& Felmate, 1992; Johnson et al., 1993; Death \& Winterbourn, 1995;
Courtemanch, 1996; Paaby et al., 1998), esto se debe en gran parte a que tienen diferentes nichos ecológicos, encontrándose así más abundantes en ciertos microhabitats que en otros. Por ejemplo, en un paquete de hojas la diversidad de táxones es mayor en comparación a un banco de arena (Maue \& Springer, 2008), y la probabilidad de incluir paquetes de hojas, y por consiguiente nuevos táxones, será más alta al aumentar el tiempo y área de muestreo (Courtemanch, 1996; Bonada et al., 2002). Segundo, la densidad de organismos en una comunidad también afecta la riqueza de táxones de una muestra, porque es posible recolectar un alto número de individuos sin acumular muchas especies (Resh \& Rosenberg, 1984; Townsend, 1989; Courtemanch, 1996). 
A

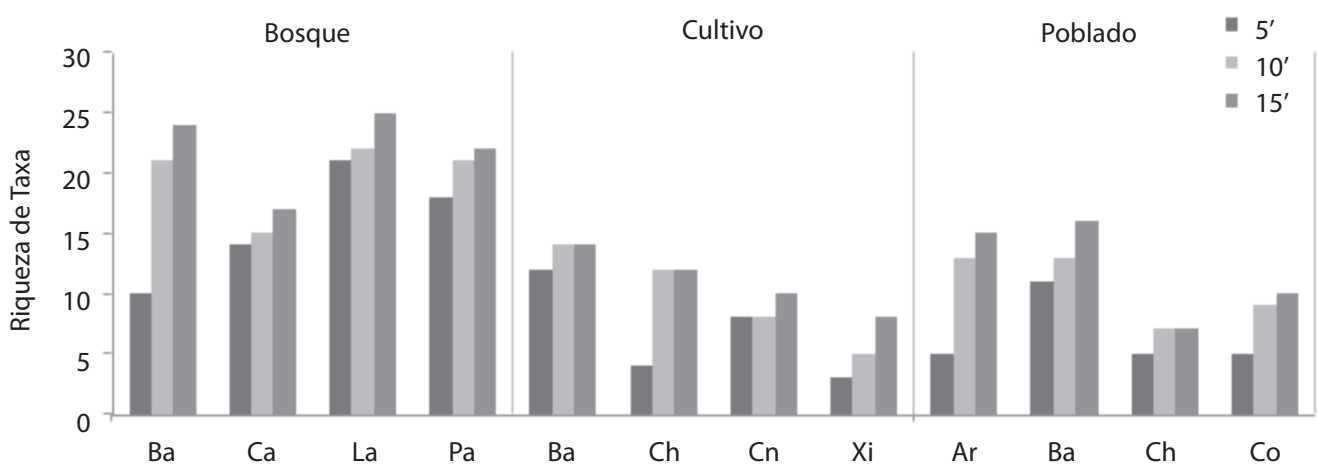

B

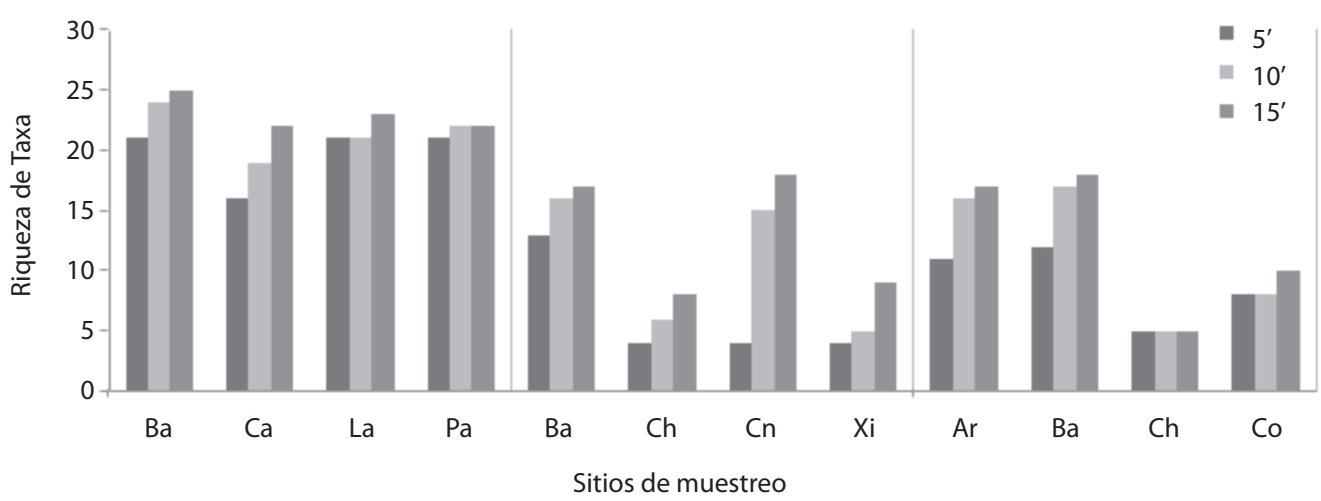

Fig. 3. Número total de táxones recolectados según el esfuerzo de muestreo en la cuenca del Lago de Atitlán, Guatemala. (A) Febrero y (B) Abril. (Ver Fig.1 para acrónimos de los sitios).

Fig. 3. Total number of táxones collected by the sampling effort in the Lago Atitlán watershed, Guatemala. (A) February and (B) April. (See Fig.1 for acronyms of the sites).

Por ejemplo, en un río alterado puede haber por unidad de muestreo una alta densidad de organismos tolerantes y la mayoría de estos organismos pueden corresponder a un mismo taxón, y por consiguiente la riqueza de táxones será baja (Townsend, 1989; Williams \& Felmate, 1992; Courtemanch, 1996).

Las especies raras también se deben considerar en este tipo de estudios. Un taxón es considerado raro si su abundancia relativa es baja dentro de la comunidad. Los táxones raros pueden ser componentes importantes en la integridad de una comunidad, debido a que algunos tienen nichos especializados y/o tolerancia a ciertas perturbaciones. Las especies raras, aunque son poco abundantes y frecuentes, pueden ser la clave para determinar los efectos de las perturbaciones a un nivel más detallado, normalmente están caracterizados por presentar rangos ecológicos muy específicos (Cao, Larsen \& Thorne, 2001; Bonada et al., 2002; Stein, Springer \& Kohlmann, 2008).

El incremento del valor del índice BMWP/ Atitlán en algunos sitios de muestreo $(\mathrm{CaBs}$, LaBs, $\mathrm{PaBs}, \mathrm{BaCu}, \mathrm{ChCu}, \mathrm{CnCu}, \mathrm{ChPo}$ y $\mathrm{CoPo}$ ) asociado con el esfuerzo de muestreo no afectó la categoría de calidad de agua de los sitios. Los pequeños cambios del valor del índice BMWP/Atitlán respecto al tiempo de esfuerzo, se podría atribuir a los táxones raros. Los individuos que son recolectados en los siguientes $5 \mathrm{~min}$, podrían contener aquellas 
A

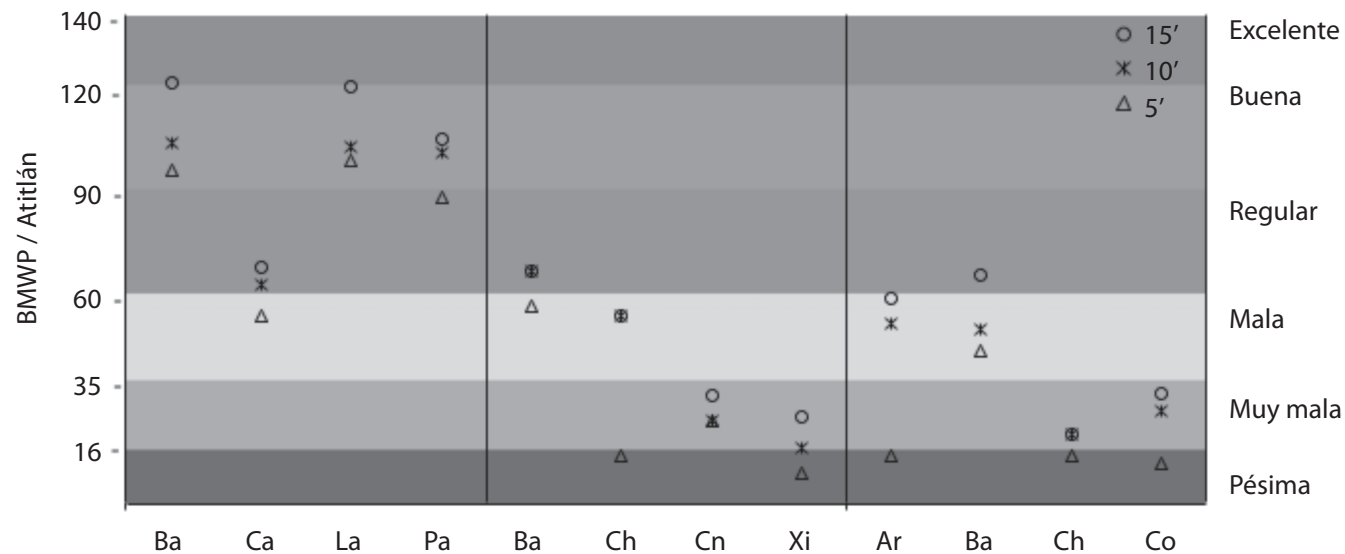

B

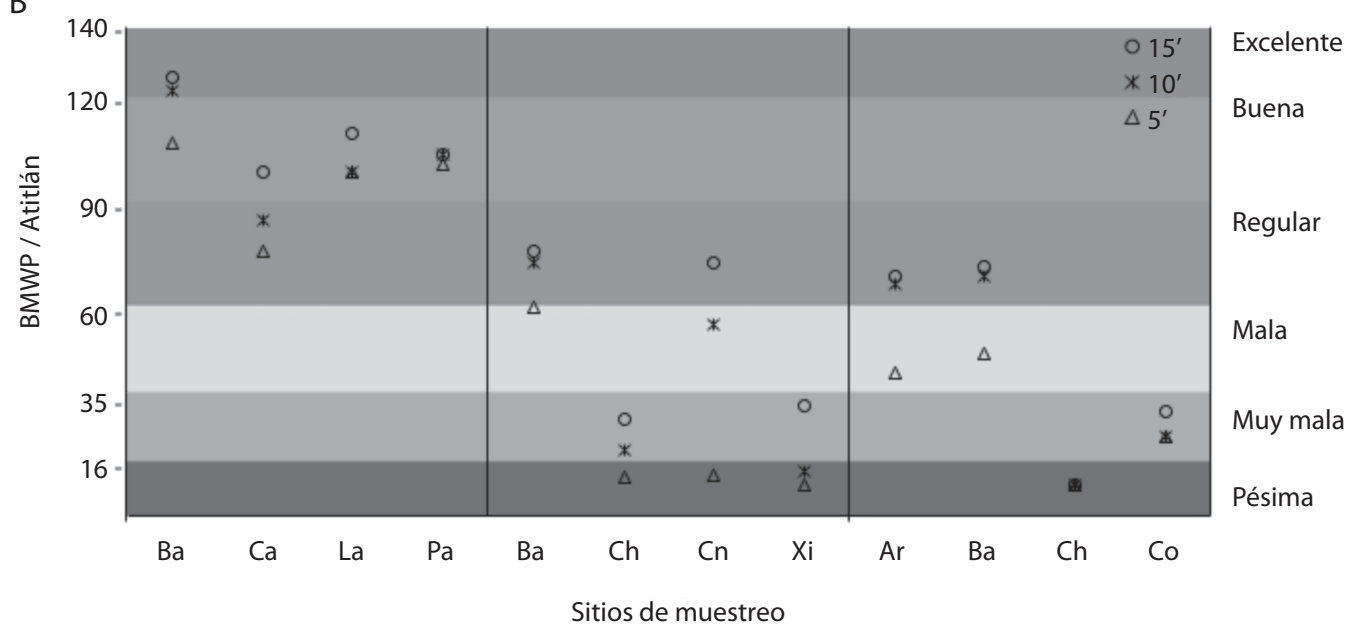

Fig. 4. Representación gráfica de los valores del índice BMWP/Atitlán por esfuerzo de muestreo. (A) Febrero y (B) Abril. (Ver Fig.1 para acrónimos de los sitios).

Fig. 4. Graphical representation of the index values BMWP/Atitlan by sampling effort. (A) February and (B) April. (See Fig.1 for acronyms of the sites).

familias raras que incrementarían el valor del índice biológico, como la familia Cordulegastridae que tiene una puntuación de 8 , y se considera una familia rara (Courtemanch, 1996; Cao \& Williams, 1999; Bonada et al., 2002; Springer et al., 2010).

Finalmente, hay que tomar en consideración que el muestreo de macroinvertebrados acuáticos con red $\mathrm{D}$ tiene el inconveniente de que se acumula una gran cantidad de material dentro de la red como hojarasca, arena y piedras (Wantzen \& Rueda-Delgado, 2009; Ramírez, 2010; Sermeño et al., 2010b). La acumulación de este material crea un reflujo interno, ocasionando así que se pierda parte del material y por consiguiente algunos organismos recolectados (Wantzen \& Rueda-Delgado, 2009; Ramírez, 2010). En este estudio como no se observó una diferencia significativa en el cálculo del Índice BMWP/Atitlán y de la riqueza de táxones registrada con un esfuerzo de muestreo de 10 y 15 minutos, se propone 
disminuir el esfuerzo de muestreo por sitio. Esta reducción de tiempo de recolecta disminuirá la cantidad de material acumulado en la red, y por consiguiente, el tiempo que se invierta en la separación e identificación del material en el laboratorio (Paaby et al., 1998; Maue \& Springer, 2008; Stein, Springer \& Kohlmann, 2008). Sin embargo, para asegurar que se obtendrá una muestra representativa de cada sitio de estudio, se recomienda mantener el mismo número de submuestras (tres) e incluir la mayor cantidad de microhabitats posibles durante el muestreo.

\section{AGRADECIMIENTOS}

Este trabajo fue realizado gracias al financiamiento y apoyo otorgado por el programa de servicio de intercambio académico alemán (DAAD) y a la Asociación Vivamos Mejor, para realizar el trabajo de tesis de la primera autora para optar al grado de M.Sc. en Biología en la Universidad de Costa Rica. Agradecemos la Universidad de Costa Rica, al Herbario USCG, a la Universidad Rafael Landívar, a la Universidad San Carlos de Guatemala, al Consejo Nacional de Áreas Protegidas, a la Universidad del Valle de Guatemala y a diversas personas que sin su aporte y ayuda no habría sido posible la realización de este trabajo. Finalmente a Gerardo Umaña, Yamileth Astorga, Bernald Pacheco y a los revisores por sus comentarios.

\section{RESUMEN}

Los macroinvertebrados son los organismos más utilizados para determinar el estado de salud de los ecosistemas en estudios de calidad del agua y biomonitoreo acuático. No obstante, los métodos para monitoreo y tiempos de recolecta no han sido efectivamente adaptados a ambientes acuáticos tropicales. En la cuenca del Lago de Atitlán, Guatemala, se evaluaron 12 ríos con diferentes tiempos de recolecta, durante la temporada seca. El método consistió en la recolecta de material orgánico e inorgánico incluyendo organismos bénticos, en diferentes microhabitats, por un periodo de tiempo $(5,10,15 \mathrm{~min})$ con una red " $\mathrm{D}$ " y preservados en etanol al 95\%; la separación y clasificación se realizó posteriormente en el laboratorio. Como se esperaba, los análisis de datos revelaron que la abundancia y riqueza de taza es mayor al incrementar el esfuerzo de muestreo. Las categorías de calidad del agua obtenidas del cálculo del índice biótico BMWP/Atitlán variaron con el esfuerzo de muestreo. Sin embargo, el análisis de Kruskall-Wallis no mostró diferencias significativas entre las categorías obtenidas con el índice y el número de táxones recolectados en los 10 y $15 \mathrm{~min}$. Por lo tanto, recomendamos que el tiempo mínimo de recolecta puede ser de 10 minutos, siempre y cuando se mantenga el mismo número de submuestras (tres) para que se incluya la mayor variedad posible de micro-hábitats durante el muestreo.

Palabras clave: biomonitoreo acuático, macroinvertebrados acuáticos, calidad del agua, Lago de Atitlán, Guatemala.

\section{REFERENCIAS}

Barbour, M. T., Gerritsen, J., Snyder, B. D., \& J. B. Stribling. (1999). Rapid bioassessment protocols for use in streams and wadeable rivers: periphyton, benthic macroinvertebrates and fish. EPA 841-B-99-002. Washington, DC: U.S. Environmental Protection Agency, Office of Water.

Bonada, N., Prat, N., Munné, A., Plans, M., Solá, C., Álvarez, M., Pardo, I., Moyà, G., Ramon, G., Robles, S., Avilés, J., Suárez, M., Toro, M., Vidal-Abarca, M., Mellado, A., Moreno, J., Guerrero, C., Vivas, S., Casas, J., Sánchez-Ortega, A., Jáimez-Cuéllar, P., Ortega, M., \& Alba-Tercedor, J. (2002). Intercalibración de la metodología GUADALMED. Selección de un protocolo de muestreo para la determinación del estado ecológico de los ríos mediterráneos. Limnetica, 21, 13-33.

Bonada, N., Dallas, H., Rieradevall, M., Prat, N., \& Day, J. (2006). A comparison of rapid bioassessment protocols used in 2 regions with mediterranean climates, the Iberian Peninsula and South Africa. Journal of the North American Benthological Society, 25, 487-500.

Cao, Y., \& Williams, D. D. (1999). Rare species are important in bioassessment (Reply to the comment by Marchant). Limnology and Oceanography, 44, 1840-1841.

Cao, Y., Larsen, D. P., \& Thorne, R. (2001). Rare species in multivariate analysis for bioassessment: some considerations. Journal of the North American Benthological Society, 20, 144-153.

Castellanos, E., Girón, N., Álvarez, M., López, M., \& España, M. (2002). Calidad del agua del Lago de Atitlán, Guatemala. Guatemala, Guatemala: Universidad del Valle de Guatemala (UVG).

Consejo Nacional de Áreas Protegidas -CONAP. (2007). Plan maestro de la reserva de uso múltiple cuenca del Lago de Atitlán. Guatemala, Guatemala: Serviprensa, S.A. 
Consejo Nacional de Áreas Protegidas -CONAP. (2008). Guatemala y su biodiversidad. Un enfoque histórico, cultural, biológico y económico. Guatemala, Guatemala: Serviprensa, S.A.

Courtemanch, D. L. (1996). Commentary on the subsampling procedures used for rapid bioassessments. Journal of the North American Benthological Society, 15, 381-385.

Death, R. G., \& Winterbourn, M. J. (1995). Diversity patterns in stream benthic invertebrate community: the influence of habitat stability. Ecology, 5, 1446-1460.

Johnson, R. K., Wiederholm, T., \& Rosenberg, D. M. (1993). Freshwater biomonitoring using individual organisms, populations and species assemblages of benthic macroinvertebrates. In D. M. Rosenberg \& V. H. Resh (Eds.), Freshwater biomonitoring and benthic macroinvertebrates (pp. 40-157). New York: Chapman \& Hall.

Maue, T., \& Springer, M. (2008). Effect of methodology and sampling time on the taxa richness of aquatic macroinvertebrates and subsequent changes in the water quality index from three tropical rivers, Costa Rica. Revista de Biología Tropical, 56, 257-271.

Merritt, R. W., Cummins, K. W., \& Berg, M. B. (2008). An introduction to the aquatic insects of North America. Iowa: Kendall/Hunt Publishing Company.

MINAE-S. (2007). Decreto $\mathrm{N}^{\circ}$ 33903. Reglamento para la evaluación y clasificación de la calidad de cuerpos de aguas superficiales. San José, Costa Rica: La Gaceta 178.

Paaby, P., Ramírez, A., \& Pringle, C. M. (1998). The benthic macroinvertebrate community in Caribbean Costa Rican streams and the effect of two sampling methods. Revista de Biología Tropical, 46, 185-199.

Quinn, G., \& Keough, M. (2003). Experimental design and data analysis for biologists. Cambridge: Cambridge University Press.

Ramírez, A. (2010). Métodos de recolección. In M. Springer, A. Ramírez \& P. Hanson (Eds.), Macroinvertebrados de agua dulce de Costa Rica I (pp. 41-50). Revista de Biología Tropical, 58(Supl 4).

Resh V. H., \& Rosenberg, D. M. (Eds.). (1984). The ecology of aquatic insects. New York: Praeger Publishers.

Resh, V. H., \& Jackson, J. K. (1993). Rapid assessment approaches to biomonitoring using benthic macroinvertebrates. In D. M. Rosenberg \& V. H. Resh (Eds.), Freshwater biomonitoring and benthic macroinvertebrates (pp. 195-233). New York: Chapman \& Hall.

Resh, V. H., Norris, R. H., \& Barbour, M. T. (1995). Design and implementation of rapid assessment approaches for water resource monitoring using benthic macroinvertebrates. Austral Journal of Ecology, 20, 108-121.

Reyes-Morales, F. (2012). Uso de macroinvertebrados acuáticos como indicadores biológicos de la calidad del agua en la cuenca del Lago Atitlán, Guatemala. (Tesis de Maestría). Universidad de Costa Rica, San José, Costa Rica.

Rosenberg, D. M., \& Resh, V. H. (Eds.). (1993). Freshwater biomonitoring and benthic macroinvertebrates. New York: Chapman \& Hall.

Sermeño-Chicas, J., Cervantes, L., Springer, M., Paniagua, M., Pérez, D., Rivas, A., Menjívar, R., Bonilla, B., Carranza, F., Flores, J., Gonzáles, C., Gutiérrez-Fonseca, P.E., Hernández, M., Monterrosa, A., \& Arias, A. (2010a). Determinación de la calidad ambiental de las aguas de los ríos de El Salvador, utilizando invertebrados acuáticos: Índice biológico a nivel de familias de invertebrados acuáticos en El Salvador (IBF-SV-2010). San Salvador: Editorial Universitaria.

Sermeño-Chicas, J., Pérez, D., Muños, S., Serrano, L., Rivas, A., \& Monterrosa, A. (2010b). Metodología estandarizada de muestreo multi-hábitat de macroinvertebrados acuáticos mediante el uso de la red " $D$ " en rios de El Salvador. San Salvador: Editorial Universitaria.

Springer, M. (2010). Biomonitoreo acuático. In M. Springer, A. Ramírez \& P. Hanson (Eds.), Macroinvertebrados de agua dulce de Costa Rica I (pp.53-59). Revista de Biología Tropical, 58, (Supl 4).

Springer, M., Ramírez, A., \& Hanson, P. (Eds.). (2010). Macroinvertebrados de agua dulce de Costa Rica I. Revista de Biología Tropical, 58(Supl 4).

Stein, H., Springer, M., \& Kohlmann, B. (2008). Comparison of two sampling methods for biomonitoring using macroinvertebrates in the Dos Novillos River, Costa Rica. Ecological Engineering, 34, 267-275.

Townsend, C. R. (1989). The patch dynamic of stream community ecology. Journal of the North American Benthological Society, 1, 36-50.

Vinson, M. R., \& Hawkins, C. P. (1996). Effects of sampling area and subsampling procedure on comparisons of taxa richness among streams. Journal of the North American Benthological Society, 15, 392-399.

Wantzen, K., \& Rueda-Delgado, G. (2009). Técnicas de muestreo de macroinvertebrados bentónicos. In E. Domínguez \& H. R. Fernández (Eds.), Macroinvertebrados bentónicos sudamericanos: sistemática y biología (pp. 17-45). Tucumán: Fundación Miguel Lillo.

Williams, D. D., \& Feltmate, B. W. (1992). Aquatic insects. C.A.B. International. Oxon: Redwood Press Ltd. 
